\title{
Comparison of the Effect of Insole Correction on Three-Dimensional Knee Joint Moments during Gait in Standing Workers with Leg Length Discrepancy
}

\author{
Yongwook Kim, PT, Ph.D \\ Department of Physical Therapy, College of Medical Sciences, Jeonju University
}

Background Few studies have been performed to investigate the correction of leg length discrepancy (LLD) on the kinetic characteristics of the knee joint using a shoe insole during gait among those who must stand while working.

Purpose To verify three-dimensional (3D) knee joint moments using a 3D motion capture system and force platforms with and without insoles during gait in standing workers with LLD.

Study design This was a repeated-measures design.

Methods Twenty-seven standing workers with LLD participated in this study. Kinetic data of the knee joint were collected using a motion analysis system and two force platforms. The participants were asked to walk freely along a 6-m walkway wearing a standard pair of shoes or standard shoes with an LLD-corrected insole. Repeated-measures analysis of variance (ANOVA) was used to compare the knee joint moments according to shoe conditions and limb sides.

Results There were significant differences in most moment variables related to the knee joint in participants with LLD-corrected insoles worn on the short limb except for the knee moments of adduction, abduction, and internal rotation $(p<0.05)$. Significant differences were shown in the maximal moment of knee adduction, abduction, internal rotation, and external rotation between the long and short legs when the participants wore standard shoes only during gait $(p<0.05)$.

Conclusions The results demonstrated a significant association between the imbalance of the knee moments and LLD among retail staff who must stand while working. Therefore, the results of this study will be useful in explaining the incidence and biomechanical characteristics of knee osteoarthritis in standing workers with LLD.

\author{
J Musculoskelet \\ Sci Technol \\ 2021; 5(1): 21-26 \\ Published Online \\ Jun 30, 2021 \\ pISSN 2635-8573 \\ eISSN 2635-8581
}

Article History Received 23 Mar 2021 Revised 3 Apr 2021 (1st) Revised 5 Apr 2021 (2nd) Accepted 5 Apr 2021

\section{CONTACT}

ptkim@jj.ac.kr Department of Physical Therapy, College of Medical Sciences, Jeonju University

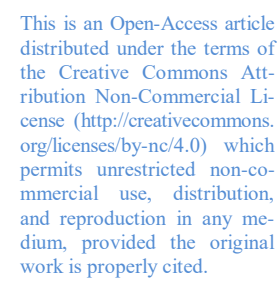

This is an Open-Access article distributed under the terms of ribution Non-Commercial $\mathrm{Li}$ cense (http://creativecommons. org/licenses/by-nc/4.0) which mercion work is properly cited.

Key words Insole; Knee moment; Leg length discrepancy; Motion analysis; Standing workers.

\section{INTRODUCTION}

Leg length discrepancy (LLD) is defined as the difference in leg length from the navel to the left and right medial (malleolus) in the supine position and is reported to negatively affect musculoskeletal posture and normal gait patterns. ${ }^{1-3}$ In addition, LLD increases asymmetrical changes in kinetic variables such as joint moment, joint force, and ground repulsion generated and kinematic variables such as joint motion range, joint angular velocity, and gait cycle in both legs during gait. ${ }^{4,5}$

LLD is caused by a wide variety of factors, ${ }^{6}$ and is classified into structural LLD and functional LLD based on the cause. ${ }^{7}$

Structural LLD occurs due to congenital factors such as hip dislocation or unilateral muscle atrophy, and functional LLD is caused by an imbalance of the lower extremity muscles due to asymmetric postural habits. ${ }^{8}$ It has been reported that about $23 \%$ of adults have an LLD of $1 \mathrm{~cm}$ or more. ${ }^{9}$ Although LLD is a common musculoskeletal pro- 
blem, it has been reported that most LLDs do not negatively affect daily life such as walking or running and do not affect the kinematic variables in the sagittal plane and walking parameters during gait. ${ }^{10,11}$ In contrast, it has been reported that individuals with an LLD of $2.5 \mathrm{~cm}$ or less had knee osteoarthritis, functional scoliosis, chronic low back pain, and plantar fasciitis. ${ }^{12-14}$

Abnormal moments and movement patterns occurring in the knee joint are highly correlated with the cause of knee osteoarthritis or chronic pain, and it has been reported that the development of abnormal kinetic variables in the knee joint and LLD are not irrelevant. ${ }^{2,15,16}$ The objective and quantitative verification of the biomechanical effects of LLD on the musculoskeletal system of the knee joint is a very important issue in managing and preventing various knee joint dysfunctions that can be caused by LLD.

The evidence base for the indications for therapeutic interventions for LLD is poor and only informal recommendations are available. ${ }^{17}$ The clinical management of LLD includes conservative physical therapy, shoe insoles, and surgical treatment. ${ }^{17}$ Among those, nonoperative care such as shoe insoles or an elevated shoe to correct the shorter leg length is always preferred for a minor to moderate LLD. ${ }^{9,17}$ Previous studies verifying the effects of insole correction in standing workers with LLD used pain intensity measured on a visual analog scale or a paper-based questionnaire to measure outcomes. ${ }^{18,19}$ Few studies, however, have explored the effect of the insole correction of LLD on the kinetic characteristics of the knee joints using a three-dimensional (3D) motion analysis system during gait among those who must stand. Thus, the purpose of this study was to investigate the effect of a shoe insole for LLD on the kinetics of the knee joints and contributions to knee stability in standing workers with LLD using a motion analysis system and force platforms during gait. It was hypothesized that when wearing the shoe insole conditions, the participants would walk with an increased symmetry of knee moments compared to without an insole during gait.

\section{METHODS}

\section{Participants}

The subjects were 27 standing workers (8 males, 19 females) with LLD. All participants had worked for at least three years and worked an average of at least eight hours per day, five days a week. The subjects were in good general health and had no problems performing laboratory walking tasks. The exclusion criteria of the study participants were those who had osteoarthritis of the knee, musculoskeletal or nervous system disorders of the lower extremi- ties, or were taking drugs that interfered with walking. Leg length was evaluated using a tape measure between the navel and both medial malleoli with the participant in the supine position. The average LLD and general characteristics of the participants are shown in Table 1. The average LLD of all participants was $12.0 \mathrm{~mm}$, while $62 \%$ of the participants had an LLD of at least $15 \mathrm{~mm}$. All participants provided written consent prior to the start of the experiment.

\section{Motion analysis}

A Vicon Motion Analysis System (Vicon Inc., Oxford, England) with eight cameras (T10 model) and two force platforms (AMTI, Watertown, MA, USA) was used to obtain 3D kinetics data of the knee joints (Figure 1). The sampling rate of the camera system was $100 \mathrm{~Hz}$ while the participants walked along a 6-m walkway. The force platforms were set in the middle of the walkway and sampled data at $500 \mathrm{~Hz}$. A calibration T-shaped wand (750 $\mathrm{mm}$ ) was used to calibrate the motion capture system and provide the $\mathrm{X}-\mathrm{Y}-\mathrm{Z}$ lab origin point in space. The Nexus 1.8.5 software program (Vicon Inc., Oxford, England) was used to process the captured kinetic data of the knee joints.

Following data acquisition and processing using the motion capture system and Nexus software, the final kinetic results and graphical reports of the knee joints were processed using the Visual3D analysis software program (C-Motion, Rockville, MD, USA). The kinetic data used in processing the moment values of the knee joints were lowpass filtered with a fourth-order Butterworth filter and a cutoff frequency of $15 \mathrm{~Hz}$. The kinematic data used to identify the exact gait cycle were low-pass filtered with a fourth-order Butterworth filter and a cutoff frequency of 6 Hz. The X-Y-Z Cardan sequence defined the order of the rotations, following the right-hand rule for the segment coordinate system axes.20 The related joint angles and knee

Table 1. Characteristics of the study participants $\quad(\mathrm{N}=27)$

\begin{tabular}{|cc}
\hline Characteristics & Mean \pm SD \\
\hline Gender $(\mathrm{M} / \mathrm{F})$ & $8 / 19$ \\
\hline Age (year) & $36.4 \pm 5.1$ \\
\hline Height $(\mathrm{cm})$ & $161.3 \pm 6.7$ \\
\hline Weight $(\mathrm{kg})$ & $61.4 \pm 8.3$ \\
\hline Leg length discrepancy $(\mathrm{mm})$ & $12.0 \pm 7.7$ \\
\hline Gait speed $(\mathrm{m} / \mathrm{s})$ & $1.2 \pm 0.3$ \\
\hline Step length $(\mathrm{cm})$ & $119.7 \pm 8.9$ \\
\hline Step width $(\mathrm{cm})$ & $9.09 \pm 2.1$ \\
\hline
\end{tabular}


moments were calculated using Visual3D software and created virtual bone segments in the walkway based on a set of reflective markers (Figure 2). The knee joint moments were normalized to body weight and height.

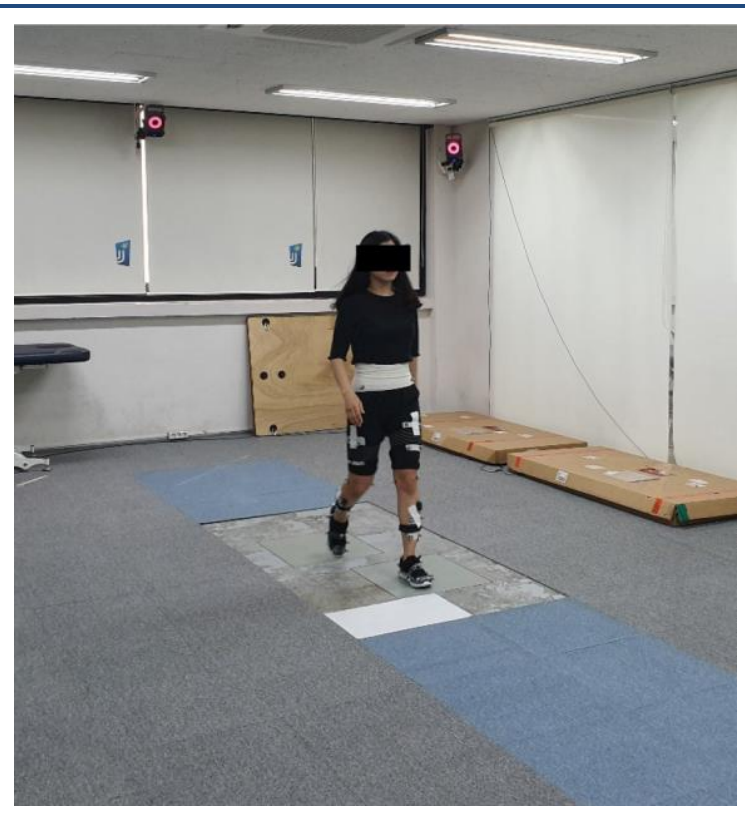

Figure 1. Three-dimensional motion analysis system and two force platforms in a $6 \mathrm{~m}$ walkway.

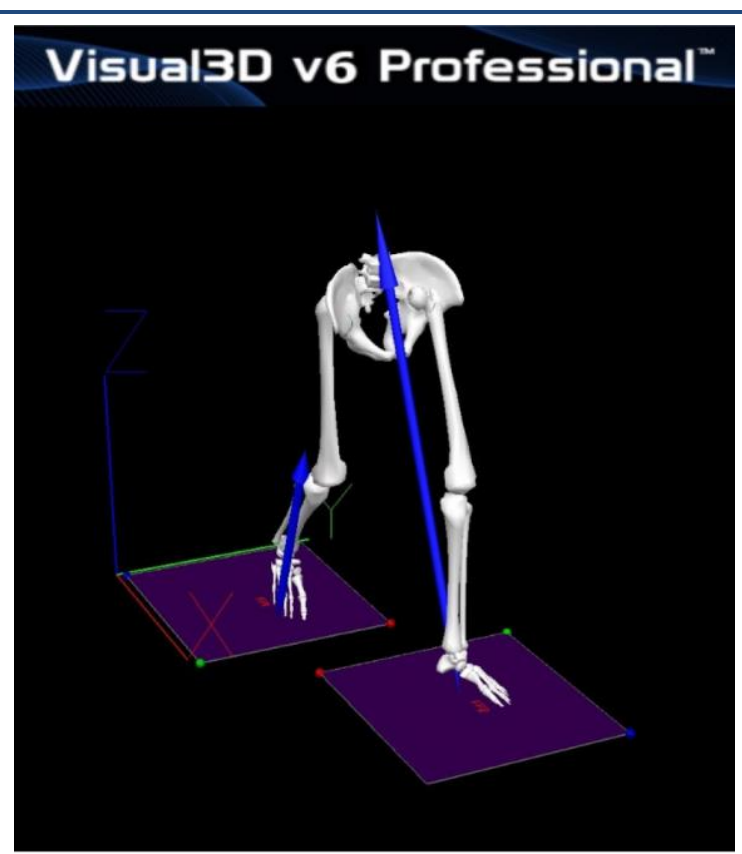

Figure 2. Calibrated anatomical system technique, used to measure the kinetic moments of knee with the Visual3D program.

\section{Procedure}

The participants were given a standard pair of shoes (Cubio, JKGOLD, Korea) for both legs and a raised insole (Cubio, JKGOLD, Korea) to correct the shorter leg. The LLD-corrected insole was made of soft polyurethane material and was made to fit each participant's shoe according to their foot size in advance. The participants were asked to walk freely along a 6-m walkway while wearing a standard pair of shoes or a pair of shoes with an LLD-corrected insole while kinetic data of the knee joints were acquired using the Calibrated Anatomical System Technique after static calibration capture. For the dynamic walking trials, the participants wore 40 retro-reflective markers $(1.4 \mathrm{~cm})$, which were attached bilaterally to the their anterior and posterior superior iliac spines, greater trochanters, femur epicondyles, malleoli, rear-feet, mid-feet, and forefeet. Four-marker clusters were attached bilaterally to the thigh and lower leg segments according to the six-degrees-offreedom (6DOF) model (Figure 1). ${ }^{20}$ First, static calibration data were obtained from each participant to create a hybrid model for later analysis of the 3D kinetics of the knee joints during the gait trials. The participants executed a total of eight to ten walk trials per condition. Before dynamic walking data collection in each shoe condition, the participants were asked to walk for 2 min to familiarize themselves with the new shoe condition. The order in which the shoes were worn (standard shoe or LLD-corrected shoe) was randomly assigned before the experimental trials began. The participants were allowed to rest for $3 \mathrm{~min}$ to avoid becoming fatigued between the rounds of data collection in the experimental trials.

\section{Statistical analysis}

The Shapiro-Wilk test was used to test for normal distribution. Repeated-measures analysis of variance (ANOVA) with Bonferroni adjustment was used to compare the kinetic data of the knee joints according to the shoe conditions and limb sides. If the main effect (shoe condition or side) was significant, post-hoc testing was used to determine the differences in the ANOVA results. All analyses were conducted using SPSS version 26.0 (IBM, Armonk, NY, USA). The level of significance was set at $p<0.05$.

\section{RESULTS}

Table 2 shows the maximal moments of each knee side (longer leg or shorter leg) according to LLD-corrected insole conditions during gait. There were significant differences in most moment variables related to the knee joint in both legs with vs. without LLD-corrected insoles applied in 
Table 2. Comparison of maximal moment of knee joint according to LLD-corrected conditions in the stance phase during gait

$(\mathrm{N}=27)$

\begin{tabular}{ccccc}
\hline Sides & Maximal knee moment (Nm/kg) & LLD-corrected shoe & Standard shoe only & $p$ \\
\hline Shorter leg & Flexion & $0.38 \pm 0.02$ & $0.31 \pm 0.03^{\dagger}$ & $0.014^{*}$ \\
Extension & $0.49 \pm 0.08$ & $0.77 \pm 0.08^{\dagger}$ & $0.002^{*}$ & $0.007^{*}$ \\
Adduction & $0.67 \pm 0.06$ & $0.95 \pm 0.09^{\dagger}$ & 0.231 \\
Abduction & $0.16 \pm 0.04$ & $0.15 \pm 0.03^{\dagger}$ & 0.242 \\
Internal rotation & $0.07 \pm 0.00$ & $0.08 \pm 0.00$ & 0.347 \\
External rotation & $0.06 \pm 0.00$ & $0.06 \pm 0.00$ & $0.012^{*}$ \\
Flexion & $0.36 \pm 0.03$ & $0.42 \pm 0.02$ & $0.002^{*}$ \\
Extension & $0.41 \pm 0.07$ & $0.19 \pm 0.02$ & $0.004^{*}$ \\
Adduction & $0.62 \pm 0.07$ & $0.32 \pm 0.08$ & 0.581 \\
Abduction & $0.17 \pm 0.05$ & $0.18 \pm 0.04$ & $0.09 \pm 0.01$ & 0.552 \\
\hline
\end{tabular}

* Significant differences between with and without LLD-corrected insole condition.

${ }^{\dagger}$ Significant differences between longer and shorter legs in without LLD-corrected condition.

${ }^{\dagger} p<0.05$ using $2 \times 2$ repeated measures analysis of variance (ANOVA) with Bonferroni's correction.

LLD, leg length discrepancy.

both legs except for the moments of abduction, internal and external rotation $(p<0.05)$. Significant differences were seen in the maximal moment of knee flexion, extension, adduction, and abduction between the long and short legs when the participants wore standard shoes without correction during gait $(p<0.05)$ (Table 2). There were no interactive effects of the maximal moment of the knee joint between the leg sides and correction conditions (with or without) $(p>0.05)$.

\section{DISCUSSION}

This study investigated 3D knee moments using kinetic data during gait with or without LLD correction using insoles in workers who must stand while working. The results of the study showed significant differences in many knee moment variables in the without-LLD-corrected insole condition compared to when wearing insole lifts for correcting LLD. In addition, the results showed that there were significant differences in many moment variables in the knee joint of the longer leg compared to the shorter leg in the without-LLD-corrected condition. The knee moment variables in the sagittal and frontal plane between the longer and shorter leg sides showed significant differences in the without-LLD-corrected insole condition during gait. The knee moment showed the first adduction and extension moments peak at a time of around $25 \%$ of the stance phase and a second adduction and flexion moments peak at $75 \%-$ $100 \%$ of the stance phase during gait. In this study, despite the presence of LLD among the standing workers who participated in this study, there were no significant differences in the transverse plane moments of the knee joint in both LLD insole conditions during gait. This is most likely because the imbalance of the knee moment and compensation control for LLD mainly occurs in the frontal plane. ${ }^{3,21}$ The maximal knee moments showed significant differences in flexion, extension, and adduction with vs. without LLD correction. In contrast, there were no significant differences in knee abduction, internal rotation, and external rotation moments with vs. without LLD correction. These results are because kinetic factors such as step length and muscle strength, which can affect knee moment, vary depending upon leg length, and the moment compensation caused by LLD may occur in lower limb joints other than the knee joint.

A few previous studies have provided objective and quantitative results of the changes in kinetic data of the knee joint according to LLD-corrected insole conditions among standing workers. Seeley et al. ${ }^{11}$ reported that an LLD of between $1.0 \mathrm{~cm}$ and $2.3 \mathrm{~cm}$ showed a significantly asymmetrical gait pattern in the knee joint moments compared to an LLD of less than $1 \mathrm{~cm}$. Zhang et al. ${ }^{22}$ also 
reported that LLD-corrected insoles or the method of raising the heel height decreased the activation of knee abductors and increased the knee extensors based on the joint kinetics. Although LLD is very common in the general population, an LLD of less than $2 \mathrm{~cm}$ did not adversely affect the musculoskeletal system or adversely affect kinematic variables such as gait. ${ }^{10,11}$ In contrast, even an LLD of less than 2 $\mathrm{cm}$ can cause chronic low back pain, functional scoliosis, knee osteoarthritis, and plantar fasciitis. ${ }^{12,14,23}$ Overall, the previous studies demonstrated a greater need to assess the effects of LLD on joints and segment stability using objective and quantitative evaluations in each experimental condition. Therefore, the advantage of this study was that it verified the biomechanical changes in the knee joint through an objective and quantitative evaluation method by applying LLD-corrected insoles to standing workers with LLD. Most of the participants in this study had an LLD of more than $15 \mathrm{~mm}$. Although this study was not intended to investigate the weight distribution of both limbs when wearing an LLD-corrected insole, when a standard shoe was used on both feet, the shorter limb bore $68 \%$ of the body weight. However, when applying the LLD-corrected insole condition, the shorter limb bore $53 \%$ of the body weight. The results indicated that the weight load proportion improved in both limbs when the LLD was corrected.

The development of an LLD and knee osteoarthritis was significantly correlated with the loss of articular cartilage in the knee joint. ${ }^{24}$ LLD is very common in the general population and causes biomechanical changes that can overload the knee joint of the shorter leg while walking. ${ }^{2}$ Therefore, even healthy people with an LLD are more likely to develop knee osteoarthritis, which most often occurs in the shorter leg. ${ }^{10}$ Harvey et al. ${ }^{16}$ reported that LLDs of $10 \mathrm{~mm}$ or more increased the probability of knee osteoarthritis occurrence in the shorter leg through radiographic examination.

The strength of this study was the verification of the kinetic effects of an LLD-corrected insole on the knee joint through reliable and valid measurement methods in participants with LLD who work for long periods while standing. However, the study had a few limitations that should be noted. First, the number of study participants was small and there was gender ratio imbalance due to the difficulty in selecting study subjects. In addition, although this study was executed on standing workers, the study subjects were employed in specific occupations, and may not represent workers in other industries. Thus, the results of this study cannot be generalized to different types of standing workers with an LLD. Further studies are needed to investigate the correlation between knee osteoarthritis and leg length inequality and verify the effects of an LLD on 3D kinetic and kinematic data of the trunk and lower limb joints using objective and highly functional tools such as a motion analysis system.

\section{CONCLUSIONS}

The results of this study showed a significant relationship between the asymmetry of the knee moment and LLD in large store staff who must stand while working. The kinetic balances of the knee joints were significantly increased when an LLD-corrected insole was applied. Therefore, the results of this study will be useful in explaining the incidence and biomechanical characteristics of knee osteoarthritis that are common in standing workers with an LLD.

\section{Key Points}

Question Does a leg length discrepancy-corrected insole have an immediate effect on the symmetry of both knee joint moments in standing workers with leg length discrepancy?

Findings There was a significant increase in kinetic balances of the knee joints when a leg length discrepancy-corrected insole was applied to workers who must stand while working.

Meaning Leg length discrepancy-corrected insoles showed kinetic effects on the knee joint in participants who worked for long periods while standing through reliable and valid measurement methods.

\section{Article information}

Conflict of Interest Disclosures: None.

Funding/Support: This study was supported by the National Research Foundation of Korea (NRF) grant funded by the Ministry of Science and ICT (No. 2018R1C1B 5042645).

Acknowledgment: None.

Ethic Approval: The study was approved by the Institutional Review Board of Jeonju University (jjIRB-170615HR-2017-0609).

\section{REFERENCES}

1. Azizan NA, Basaruddin KS, Salleh AF. The effects of leg length discrepancy on dtability and kinematics-kinetics deviations: a systematic review. Appl Bionics Biomech. 2018;11:5156348.

2. Resende RA, Kirkwood RN, Deluzio KJ, et al. Mild leg length discrepancy affects lower limbs, pelvis and trunk biomechanics of individuals with knee osteoarthritis during gait. Clin Biomech (Bristol, Avon). 2016;38:1-7. 
3. Walsh M, Connolly P, Jenkinson A, et al. Leg length discrepancy -an experimental study of compensatory changes in three dimensions using gait analysis. Gait Posture. 2000;12(2):156-161.

4. Kim YW. Characteristics of pelvic ranges according to artificial leg length discrepancy during gait: three-dimensional analysis in healthy individuals. J Korean Soc Integrative Med. 2019;7(2):59-67.

5. Needham R, Chockalingam N, Dunning D, et al. The effect of leg length discrepancy on pelvis and spine kinematics during gait. Stud Health Technol Inform. 2012; 176:104-107.

6. Jo MJ, Kim DH, Han DW, et al. Effect of artificial leg length discrepancy on $3 \mathrm{~d}$ hip joint moments during gait in healthy individuals. PNF \& Mov. 2019;17(3):391-399.

7. Blake RL, Ferguson H. Limb length discrepancies. $J$ Am Podiatr Med Assoc. 1992;82(1):33-38.

8. Gurney B. Leg length discrepancy. Gait Posture. 2002; 15(2):195-206.

9. McCarthy JJ, MacEwen GD. Management of leg length inequality. J South Orthop Assoc. 2001;10(2):73-85.

10. Resende RA, Kirkwood RN, Deluzio KJ, et al. Biomechanical strategies implemented to compensate for mild leg length discrepancy during gait. Gait Posture. 2016; 46:147-153.

11. Seeley MK, Umberger BR, Clasey JL, et al. The relation between mild leg-length inequality and able-bodied gait asymmetry. J Sports Sci Med. 2010;9(4):572-579.

12. Raczkowski JW, Daniszewska B, Zolynski K. Functional scoliosis caused by leg length discrepancy. Arch Med Sci. 2010;6(3):393-398.

13. Defrin R, Ben Benyamin S, Aldubi RD, et al. Conservative correction of leg-length discrepancies of $10 \mathrm{~mm}$ or less for the relief of chronic low back pain. Arch Phys Med Rehabil. 2005;86(11):2075-2080.

14. Mahmood S, Huffman LK, Harris JG. Limb-length discrepancy as a cause of plantar fasciitis. J Am Podiatr
Med Assoc. 2010;100(6):452-455.

15. Noll DR. Leg length discrepancy and osteoarthritic knee pain in the elderly: an observational study. J Am Osteopath Assoc. 2013;113(9):670-678.

16. Harvey WF, Yang M, Cooke TD, et al. Association of leg-length inequality with knee osteoarthritis: A cohort study. Ann Intern Med. 2010;152(5):287-295.

17. Vogt B, Gosheger G, Wirth T, et al. Leg length discrepancy-treatment indications and strategies. Dtsch Arztebl Int. 2020;117(24):405-411.

18. Rannisto S, Okuloff A, Uitti J, et al. Leg-length discrepancy is associated with low back pain among those who must stand while working. BMC Musculoskelet Disord. 2015;16:110.

19. Rannisto S, Okuloff A, Uitti J, et al. Correction of leglength discrepancy among meat cutters with low back pain: a randomized controlled trial. BMC Musculoskelet Disord. 2019;20:105.

20. Collins TD, Ghoussayni SN, Ewins DJ, et al. A six degrees-of-freedom marker set for gait analysis: repeatability and comparison with a modified Helen Hayes set. Gait Posture. 2009;30(2):173-180.

21. Kim Y, Richards J, Lidtke RH, et al. Characteristics of clinical measurements between biomechanical responders and non-responders to a shoe designed for knee osteoarthritis. Gait Posture. 2018;59:23-27.

22. Zhang S, Clowers KG, Powell D. Ground reaction force and 3D biomechanical characteristics of walking in short-leg walkers. Gait Posture. 2006;24(4):487-492.

23. Raczkowski JW, Daniszewska B, Zolynski K. Functional scoliosis caused by leg length discrepancy. Arch Med Sci. 2010;6(3):393-398.

24. Sharma L, Eckstein F, Song J, et al. Relationship of meniscal damage, meniscal extrusion, malalignment, and joint laxity to subsequent cartilage loss in osteoarthritic knees. Arthritis Rheum. 2008;58(6):1716-1726. 